\title{
RISK MAPPING OF LANDSLIDE HAZARD ON ROAD NETWORK
}

\section{IN KOREA}

Sohee Lee $^{1}$ and Yoonjin Yoon ${ }^{2}$

${ }^{1}$ Disaster Information Research Division, National Disaster Management

Research Institute, 365 Jongga-ro, Jung-gu, Ulsan 44538, Korea

2 Department of Civil Environmental Engineering, Korea Advanced Institute of Science and Technology, 291 Daehak-ro, Yuseong-gu, Daejeon 305-701,

Korea

shlee4649@korea.kr

$+82-52-928-8222$

$+82-52-928-8209$

ORCID ID orcid.org/0000-0003-0368-7752

\section{Crossref}

http://dx.doi.org/10.26739/2433-202x

Issue DOI http:/ /dx.doi.org/10.26739/2433-202x-2017-6-6

Article DOI http:/ / dx.doi.org/10.26739/2433-202x-2017-6-6-6

This paper describes a methodology for measuring road network vulnerability due to landslide hazard in Korea using the Geographic Information System (GIS). To identify hazardous road links (or road areas) in relation to areas Physical, Chemical Science and Engineering 
susceptible to landslide, we employed overlay analysis, which integrates two map features: the road network and landslide hazard. Then, using the values of landslide hazard grade and road length (or road area) obtained by overlay analysis, we proposed a matrix analysis to assess the road network vulnerability at the aggregate level as an adjusted unit link according to landslide hazard with a consideration of two indices: one index is the seriousness of the landslide hazard grade; the other index is the proportion of road length (or road area) that is exposed to landslide hazard within an adjusted unit link. The resulting maps can be useful to decision makers with no prior knowledge of the technique. Results show different road risk maps, and also highlight vulnerable road links through the entire road network. This approach can be useful to prepare new transportation infrastructure information, manage that information, and adapt to the lack of reliable information on natural disaster hazards occurring across a geographical area.

Key words: risk mapping; road network; landslide hazard map; GIS; Korea 
1. INTRODUCTION. The breakdown of essential urban infrastructure is one of the most widely shared characteristics of all disasters. Whether partial or complete, the failure of urban infrastructure leads to preventable loss of life and damage to property. Moreover, the indirect impacts due to natural hazards spread throughout a nation, considering the interdependencies of interconnected urban infrastructures in relatively confined geographic areas (e.g., U.S. Department of Energy, 2012).

These issues have not received a great deal of attention in the research literature in general and the transportation network is no exception. When a disaster occurs, as one critical part of a nation's infrastructure, the transportation network has a central role in supporting supply delivery, rescue and emergency medical services, and evacuations at the stage of response; this network also facilitates early recovery activities at the stage of restoration. Furthermore, both indirect damage that can cause delays in emergency responses and disaster restorations, and cascading failures will be hard to predict.

From this perspective, the lack of reliable transportation network can be seen to cause major damage directly or indirectly in our lives and can make Physical, Chemical Science and Engineering 
human settlement unacceptably risky. That is why pre-disaster risk management planning, essential to post-disaster resilience, is known to be vital.

In the process of planning disaster management and response systems, risk assessment is fundamental and essential to any work on risk reduction and recovery (CEU, 2010; FEMA, 2001; FEMA, 2011). According to the referenced documents, risk mapping is necessary for the identification and assessment of risks that could lead to potential losses of people, property, systems, or other elements present in hazard zones. However, the methods in the above research are ultimately focused on the estimation of losses.

In this study, we suggest a methodology of risk mapping to identify hazardous road lines in order to propose advance preparation for the lack of reliable transportation networks; this is carried out via a process of pre-disaster management planning. Accordingly, we identify that 'hazards' come from natural disasters that cause harm or loss; a 'hazard map' is a map that portrays levels of hazard occurring across a geographical area; 'risk' is the potential for damages caused by hazards, even though this metric is unquantified and deals with the probability of occurrence; then, a 'risk map' is a map that portrays 
levels of a risk based on a hazard map. We examine landslide hazard as an example natural disaster in this study.

The aim is to develop a methodology for measuring road network vulnerability caused by landslide hazard considering the motorway network in Korea as an example. To do so, this paper describes the risk mapping of landslide hazards on the road network based on GIS spatial data. Resulting maps can be used for pre-disaster management planning to develop safer routes to emergency services or safer evacuation plans. As a preliminary step for providing pre-disaster management planning, our approach, based on GIS techniques, can be used to form a basic process to prepare for, manage, and adapt to the vulnerabilities of the transportation network to all kinds of natural hazards.

The paper is organized as follows. Section 2 reviews the literature on hazard maps and disaster risk assessment. Section 3 presents the method framework and the data used in the study. Section 4 presents the results, with Section 5 drawing conclusions and assessing the limitations of the current approach.

\section{REVIEW OF LITERATURE}


With reference to hazard mapping, the flood hazard map is the most important example in Korea because floods are the most frequent hazard; also, the damage caused by floods is the very great (NEMA, 2008-2012). Within a portal system named WAMIS (WAter Management Information System) provided by the Korean Ministry of Land, Infrastructure and Transport (MLIT), statistical and spatial data related to flooding information, such as waterlogged areas, flood water level, rainfall per hour in flood period, etc., are included. In addition, in order to manage urban streams, the RIMGIS (RIver Management GIS) system has been operated. However, no spatial data on other disasters is available. It seems that the Korean government is able to amass historical flood hazard data, but there is something lacking for natural disasters other than floods.

Central to this paper is how to assess the impact of hazards induced by natural disasters; this assessment will lead to effective strategies for reducing risk. There have been a number of studies documenting the impact of natural hazards on urban elements and the environmental, economic, and social factors inherent in different natural disasters. 
In such reports as have been compiled by the government authorities (e.g., CEU, 2010; FEMA, 2001; FEMA, 2011), to quantitatively assess the overall damage, a conceptual framework for risk assessment and basic methodology has been formulated. However, mapping is only used as a standard method for the estimation of losses, to determine the impact of damage in a given geographic area.

There have been a few domestic studies (e.g., Kim, 2003; Kim and Shin, 2009) that have determined the need for a national approach in the development of the general safety management system in Korea. For different types of urban disaster, those researchers proposed safety indicators such as flood hazard and fire hazard in buildings, but did not specify the impact on the urban infrastructure.

There are other studies that have estimated the quantitative risk due to natural hazard. A quantitative procedure for mapping landslide risk from various different perspectives has been developed (e.g., Aleotti and Chowdhury, 1999; Remondo et al., 2008). They focused on the estimation of landslide probability considering different factors; however, they did not consider impacts on urban elements, such as damage to life or property caused by landslide hazard. 
A few studies have been done related to the risk assessment of road networks exposed to natural or human disasters. Dalziell and Nicholson (2001) estimated the risk of closure of a desert road to all road users by employing the techniques of risk analysis, namely hazard identification, and risk assessment and management. Irstea and Mermet (2012) proposed a new specific methodology based on network structure analysis to assess road vulnerability under direct and indirect effects of natural phenomena. Scenna and Cruz (2005) simulated the risk distribution and its impact on people, focusing on road accidents in areas undergoing hazardous material transportation.

In the procedures of risk assessment, most studies are fully occupied with attempts to quantitatively estimate damage to life and property; that damage is generally converted into economic cost. However, estimating the quantitative damage is not simple work, and one reason for its difficulty might be that proper data sets are not always at hand to determine which variables are important. Thus, we propose a road risk map that indicates where on the road network are the highest priority areas to be managed for safety in advance of any disaster; this map will be of use to the authorities of road safety and management. 
In terms of disaster risk management, "vulnerability" is broadly defined as the potential for losses; this potential may be measured in terms of environmental, physical, or economic damage (Cardona, 2003, Cutter, 1996, Thomalla et al., 2006). However, urban infrastructure, which becomes critical when a disaster occurs, has not yet been fully assessed in terms of disaster vulnerability.

There are two ways in which this paper can be differentiated from previous studies. The first is that this paper addresses the lack of interest in vulnerability studies of the transportation network and allows the managing of vulnerable networks in advance, against damage induced by specific hazards. The second important aspect of this paper deals with the fact that a number of studies have suggested a baseline of risk assessment for all type of hazards on community, urban, and national levels; however, within the procedures of risk mapping, urban elements that are exposed to hazard need to be identified. For this, a variety of spatial data with high accuracy and reliability has to be generated. In this study, utilizing already deployed spatial data, we propose a simple way to map risk to the road network due to landslide hazard. 


\section{METHODOLOGY}

\subsection{Study Area and Data Set}

\subsubsection{Study Area}

The Republic of Korea (hereafter "Korea") is targeted as a study area. According to the population and housing census of 2010, Korea had 48.6 million people; the land area covers about 99.8 thousand square kilometers. More than three-quarters of the land is mountainous (Statistics Korea, 2010).

Table 1 presents the amount of damage, over the last 5 years, caused by natural disasters in Korea. Major types of natural disaster are typhoons and heavy rains; the amount of damage caused by those two hazards accounted for more than 80 percent of damage, except for the year 2008. The table does not include damage due to landslides, because landslides generally occur along with typhoons, heavy rain, or earthquakes (e.g., Dai et al., 2002; Sassa et al., 2004), so damage due to landslides has not been included in this record.

Disasters caused by landslides have continued to increase because the amount of rainfall and also localized torrential rainfall have steadily increased due to the effects of climate change. According to the statistics of the Korea 
IFS 2,7

Forest Service (KFS), 43 lives were lost due to landslides and the amount of damage that occurred was estimated at 147 billion KRW (approximately 147 million USD) in 2011. This record is to date the highest from damage due to landslides. In addition, a huge landslide swept away the southern part of Seoul City, causing much damage. Thus, it is necessary to evaluate the direct effects on our lives that landslides can potentially cause in order to prepare for any possible damage.

\subsubsection{Landslide Hazard Map}

Using logistic regression analysis, the Korea Forest Research Institute (KFRI) in 2012 developed a landslide hazard map of Korea. The KFRI is an affiliated agency under the Korea Forest Services (KFS); it is in charge of maintaining Korea's forest land. There are nine main landslide factors: slope degree, slope length, slope curvature, topographic index, forest floor, forest type, soil depth, bedrock, and modification factors; these are all considered in order to estimate the probability of landslide. The probability of landslide takes a value between 0 and 1, and falls into 5 grades. This landslide hazard map with 5 grades of classification is available on request, for research purposes, for researchers who are employed at research institutes in Korea. The data is 
available as a raster data set $(10 \times 10$ meter grid cells). Table 2 presents the classification of the landslide hazard grades and the number of grid cells and their proportion. Figure 1 is the landslide hazard map of Korea; it is divided into 5 landslide hazard grades. About 35\% of the grid cells are distributed between grades 3 and 4, which means they are classified as "more than highly susceptible" to landslide.

\subsubsection{Road Network of Korea}

Information on the road network, as a vector data set, is offered by the Korea Transport Database (KTDB), which is operated and managed by the Korea Transport Institute (KOTI). The road network is divided into several classes according to its function and management authority. In this study, the motorway network map, which was updated in Dec. 2011, is used (Figure 2). As a grid pattern, the motorway network links major urban areas throughout the country. The total length of motorway network is about $8,124 \mathrm{~km}$, and that consists of 7,257 links. These links are separated into different classifications of road based on their various speed limits; the classifications include intersections, U-turn spots, interchanges, etc. In this study, these links are adjusted as per the location of interchanges and junctions because, on the 
motorway, vehicles only can enter and exit through interchanges; they can

also only manoeuvre to their destinations through junctions. So, we created a risk map for the road network using the adjusted unit link between two interchanges (or junctions) for any place that had a landslide hazard or at which a landslide had occurred at a certain point on that link. Consequently, the number of links was adjusted from 7,257 to 911 links.

Moreover, in order to compare the difference between road lines and road areas when we mapped the road risk due to landslide hazard, we used buffer analysis in GIS to work into the polygon data a line that is only offered as a road network data set. Buffer analysis was used to identify areas surrounding geographic features in order to evaluate the magnitude of damage caused by landslides. Multiplying 3.5 meters by the number of lanes gave the buffered distance. More details about method will be described in the following section.

\subsection{How to Generate the Landslide Road Risk Map using GIS}

As we mentioned earlier, the purpose of this study is to suggest an alternative process to generate a landslide hazard road risk map in order to evaluate network vulnerability in large-scale transportation networks. In Physical, Chemical Science and Engineering 
doing so, the Geographic Information System (GIS) is the most common and crucially efficient tool applicable to the evaluation of a wide range of different impacts. We employed an overlay analysis application in GIS. To identify the hazardous road links (or road areas) in relation to areas susceptible to landslide, this application integrates spatial data with attribute data on two map features: the motorway network (road line or road area) and landslide hazard. In this study, we used the ArcGIS 10.0 software package for all parts of the GIS analysis and visualization.

The process of charting the landslide hazard road risk map can be summarized as follows. First, at its simplest, the motorway network (line-road link and polygon-road area) and landslide hazard (polygon) are overlaid to create a new line (or polygon) layer. As a result of this overlay analysis, the most disaggregate level of the road line (or road area) with landslide hazard grades will be measured. Next, the total length (or area) of the road of each landslide hazard grade will be calculated by selecting all lines (or polygons) within the adjusted unit link. However, at the disaggregate level, there is a difficulty in visualizing a landslide hazard road risk map, because small pieces of lines (or areas) are so small as to be indistinct. Therefore, we proposed matrix analysis to assess the road network vulnerability at the disaggregate Physical, Chemical Science and Engineering 
level as unit links adjusted by landslide hazard with a consideration of two indices: the first index is of the seriousness of the landslide hazard grade; the other index is the proportion of the road length (or area) that is exposed to landslide hazard within the adjusted unit link. Then, the landslide hazard road risk map was generated to allow the investigation of the spatial distributions of the two indices in sequence. More details on matrix analysis with these two indices will be provided in the following section.

\subsection{Two Indices for Risk Mapping of Landslide Hazard on Motorway Network}

By employing the overlay analysis that we mentioned in section 3.2, small pieces of lines (or polygons) that are at the most disaggregate level with a certain landslide grade can be obtained. In this section, in order to assess the road network vulnerability, two indices will be introduced and we will also visualize the road risk mapping due to landslide hazard at the aggregate level, which is the same as the adjusted unit link. The number of adjusted unit links is 911 . 


\subsubsection{Link Length (or Area) Weighted Average of Landslide Hazard Grade for Each Adjusted Unit Link}

In order to calculate the seriousness of the landslide hazard grade for each adjusted unit link at the aggregate level, a weighted average is used. The link length (or area) weighted average of landslide hazard (WALH) index is calculated as follows:

The weighted average of each adjusted unit link $i$ is

$$
W A L H_{i}=\frac{\sum g_{k} d_{j k}}{\sum d_{j k}}
$$

where $d_{j k}$ is the length (or area) of link $j$ with landslide hazard grade $k$, and $g_{k}$ is the landslide hazard grade. The landslide hazard grades are described as follows: grade 1 indicates areas that have low susceptibility to landslides, grade 2 indicates areas that have low susceptibility, grade 3 is highly susceptible areas, and grade 4 indicates very highly susceptible areas when causal factor such as rainfall are triggers. In addition, links at which there is no susceptibility to landslide are graded 0 . So, the values of the WALH index will be 0 or between 1 and 4 . If the value of the WALH index becomes large this means that the adjusted unit link $i$ Physical, Chemical Science and Engineering presents a very high susceptibility to 
landslides. In other words, that adjusted unit link is vulnerable to landslide hazard as compared with the other adjusted unit link which have less value of WALH index.

\subsubsection{Proportion of Link Length (or Area) that is exposed to Landslide Hazard for Each Adjusted Unit Link}

The proportion of link length (or area) that is exposed to landslide hazard for each adjusted unit link (PLLH) index is measured as follows:

The proportion of each adjusted unit link $i$ is

$$
P L L H_{i}=\frac{\sum d_{j k}}{d_{i}}
$$

where $d_{j k}$ is the length (or area) of link $j$ with landslide hazard grade $k$ and $d_{i}$ is the length (or area) of adjusted unit link $i$.The values of the PLLH index will be between 0 and 1 . If the value of the PLLH index becomes 0 this means there is a small portion of a small piece of a link that is exposed to landslide hazard within the adjusted unit link $i$; on the contrary, a large portion of links are distributed in road link $i$. 


\subsubsection{Matrix Analysis using Two Indices: the Values of WALH and PLLH}

Matrix analysis is a useful and popular method to provide a standard tool of treating the relationship between two indices in assessing the vulnerability of road networks due to landslide hazard. In this section, a risk matrix based on two indices, used for the ranking of road risk due to landslide hazard, is described.

A matrix is proposed that integrates consideration of both WALH and PLLH indices, a definition that we mentioned above, according to the landslide hazard for each adjusted unit link. In this matrix analysis, we used the $\mathrm{X}$ axis to represent the four risk levels (the value of the WALH index) with the addition of the values 'none'--; 'low', 'medium', 'high', and 'very high'--; and the $\mathrm{Y}$ axis to represent the ration levels (the value of PLLH index) with also adding the values 'none'--; 'small', 'medium', 'large', and 'very large'. The sample matrix shown below in Table 3 indicates how the overall risk levels of low, medium, high, and very high are derived.

Two indices: the weighted average of the landslide hazard (WALH index) and the proportion of the road links exposed to landslide hazard (PLLH index), are considered to indicate the risk to the road links. When the values of 
WALH and PLLH are large, generally the risk to the road links is also very high.

\section{RESULTS}

This section provides a demonstration of the proposed risk assessment method for landslide hazard in the case of the motorway network in Korea. The motorway network is selected because it is: a key national traffic network of roads connecting major cities in the country, and will be performing important functions as transport lifelines for emergency goods and heavy traffic if a national emergency situation occurs. In addition, the method that we proposed in this study can be easily applied to other structured levels of road networks.

\subsection{Risk Mapping of Landslide Hazard on Motorway Network using Two Indices}

Figure 3 presents the road risk map of landslide hazard generated by assessing the link length weighted average according to landslide hazard Physical, Chemical Science and Engineering 
grades (the value of the WALH index). Road sections are allocated into colorcoded categories from low risk to very high risk; then, there is also the category of no risk.

Table 4 shows the number of links and the proportion determined by classifying the value of the WALH index. As can be seen, more than half of the adjusted links have been exposed to landslide hazard. Moreover, approximately $20 \%$ of the total of adjusted links are classified as 'high'- and 'very high' risk due to landslide hazard.

Risk maps based on the weighted average (WALH index) do show the extent to which the adjusted unit links of the network might result in the risk being higher or lower than the average, which is aggregated at the unit link due to landslide hazard. However, this map does not show how large a portion of the adjusted unit link length might be exposed to the risk of landslide. So, a road link might not be validated as 'high risk' generally, even when its value of the WALH index is found to be 'high'. That is why, in order to evaluate the risk of the road network due to landslide hazard, we have proposed two indices, considering both landslide hazard grade and proportion of link length that is exposed to landslide hazard. 
Figure 4 shows the road landslide risk map, generated by assessing the proportion of link length that is exposed to landslide hazard within an adjusted unit link (the value of the PLLH index). Road sections are allocated into colorcoded categories, with risk levels from small to very large; there is also the category of 'none' for no risk.

Table 5 shows the number of links and the proportion determined by classifying the value of PLLH. In comparison with Table 4, it can be seen that the proportion that is classified as 'none' remains unchanged. However, less than $10 \%$ of the adjusted links of the total are classified as 'large' or 'very large'. This means that some adjusted unit links have a low value of PLLH index even though the value of the WALH index is generally high.

Figure 5 shows the road risk map for landslide hazard determined using matrix analysis that considered both of the two indices that we mentioned above. The risk of the road links is divided into 4 coloured bands from low (green) risk, medium risk (yellow), high risk (red), and very high risk (black). This adjustment is based on the combination of the weighted average of the landslide hazard and the ratio of the length of the road link that is exposed to landslide hazard to the length of the road link measured for overlay analysis of the road network and landslide hazard maps. In general terms, the concept Physical, Chemical Science and Engineering 
of 'severity' is now used to refer to the level of danger of landslide hazard; the term 'frequency' is used to refer to the degree of landslide hazard exposure within a unit road link.

Table 6 shows the number of links and the ratio obtained by classifying the road risk matrix using two indices: the values of WALH and PLLH. In comparison with Tables 4 and 5, of course, the proportion of items that are classified as 'none' remains unchanged. About $5 \%$ of adjusted links of the total are classified as 'high risk' or 'very high risk'.

\subsection{Difference of Weighted Average of Landslide Hazard Grade between Link and Polygon Data Sets of Road Network}

In general, landslide damage has a large influence on neighboring areas. To put this in perspective, there is a limit as to what kinds of base maps we can use to make a road risk map. The effect of an avalanche of earth and rocks on neighboring areas is completely disregarded in this paper because there is as yet no exact prediction map for avalanches of earth and rocks available to the public in Korea. However, if a higher quality well-predicted landslide hazard map could be obtained, it would have no effect on our methodology except to 
allow some progress in improving the accuracy of the road risk map. So, basically, how well-predicted the landslide hazard map is that we employed is not our concern because the resulting map can be changed according to how precise the base map is; however, the process of risk mapping will be the same. Another important thing is that the road network that we employed to overlay the analysis is offered as a line data set. While showing the landslide hazard map, concerning the fact that landslides have strong influences on neighboring areas, rather than using a line data set, a polygon data set of road lines suits our approach. For the reason mentioned, in this section, we make a comparison of road risk mapping processes between those process that use lines and those that use polygon data sets.

Figure 6 presents the difference of the weighted average of the landslide hazard grade between a line and a polygon data set for the road network. Shades of the two colors red and blue on the map represent the differences in the value of the WALH index between the line and the polygon data sets.

If the difference in the value is positive, this means the line data set has been found to have a value larger than that of the polygon data set. Therefore, road lines with two shades of red colour indicate that an area less susceptible to landslide is distributed surrounding a road link. Consequently, the value of Physical, Chemical Science and Engineering 
the road risk, which is the weighted average of the landslide hazard grade, is overestimated. On the contrary, if the difference in the value is negative, this means that the value of the line data set has been found to be smaller than that of the polygon data set. Therefore, road lines with two shades of blue colour indicate areas surrounding the road line that are more susceptible to landslide. Consequently, the value of the road risk is underestimated.

Table 7 shows the number of adjusted road links with their transition states when the weighted average of the landslide hazard grade is measured in comparison with the line and polygon data sets. The matrix explains how many adjusted road lines are changed to their state of road risk grades. As the table shows, most of the adjusted road links have not changed their state (about $87.9 \%$, which includes 801 of the total 911 links); the rest have changed their state (about $12.1 \%$, which includes 110 of the total 911 links). When a line data set is used to overlay the analysis, about $6.7 \%$ of the road links (61 links) are probably overestimated in terms of their risk states; on the other hand, $5.4 \%$ of the road links (49 links) are probably underestimated.

\section{CONCLUSIONS}

In this study, employing GIS techniques, we have described a method of 
risk mapping of landslide hazard on the road network of Korea. The resulting map can be useful for decision makers with no prior knowledge of the technique. Moreover, the map could be incorporated into GIS, along with other complementary data sources, in order to assist in the management of associated risks from natural hazards.

Results were used to generate different road risk maps, and also highlighted the vulnerable road links throughout the entire road network. This approach can be useful to prepare new transportation infrastructure information, manage that information, and adapt to the lack of reliable information on natural disaster hazards occurring across a geographical area.

This type of study is at the leading edge of road safety and disaster management planning in Korea. By identifying those adjusted road links classified as more than 'high-risk', road management authorities can target road safety investigations and investment at specific sections of road lines where improvements can be made that will have the most benefit from the perspective of road safety assessment against landslide hazard.

However, such maps must naturally be checked and validated with reliable procedures because the results of the overlay analysis rely on the spatial accuracy of the GIS layers. If the layers do not line up well, then the Physical, Chemical Science and Engineering 
attributes inferred by the overlay may be incorrect. Therefore, highly precise base maps are more important than anything else if we are to obtain a practical road risk map using our approach. Consequently, the results are only as good as the GIS spatial data used for the analysis.

The proposed methodology could possibly be applied to visualize the road risk due to landslide hazard in a variety of road networks that are functionally different.

\section{REFERENCES}

Aletti, P., Chowdhury, R., 1999, Landslide hazard assessment: summary review and new perspectives, Bulletin of Engineering Geology and the Environment 58, 21-44.

Cardona, O. D., 2003, The need for rethinking the concepts of vulnerability and risk from a holistic perspective: a necessary review and criticism for effective risk management, Chapter 3 of the book "Mapping Vulnerability: Disasters, Development and People", G. Bankoff, G. Frerks, D. Hilhorst (Ed), Earthscan Publishers, London. http:/ / www.lared.org/public/articulos/2003/nrcvrfhp/nrcvrfhp_ago-04-2003.pdf 
CEU (Council of the European Union), 2010, Risk assessment and mapping guidelines for disaster management, Commission Staff Working Paper SEC (2010) 1626 final, EU. Available at http://ec.europa.eu/echo/files/about/COMM_PDF_SEC_2010_1626_ F_staff_working_document_en.pdf

Cutter, S. L., 1996, Vulnerability to environmental hazards, Progress in Human Geography 20(4), 529-539.

Dai, F. C., Lee, C. F., Ngai, Y. Y., 2002, Landslide risk assessment and management: an overview, Engineering Geology 64, 65-87.

Dalziell, E., Nicholson, A., 2001, Risk and impact of natural hazards on a road network, Journal of Transportation Engineering 127(2), 159-166.

FEMA (Federal Emergency Management Agency), 2001, Understanding your risks: identifying hazards and estimating losses, State and Local Mitigation Planning How-To Guide \#2 (FEMA 386-2), USA. Available at http:/ / www.fema.gov/medialibrary/assets/documents/4241?id=1880

FEMA (Federal Emergency Management Agency), 2011, FEMA's risk mapping, assessment, and planning (risk map), Fiscal Year 2011 Report to Congress, USA. Available at http://www.fema.gov/media-library- 
data/20130726-1756-25045-9468/fy11_rm_report.pdf

Irstea, J. T., Mermet, E., 2012, Analysis of importance of road networks exposed to natural hazards, Proceedings of the AGILE'2012 International Conference on Geographic Information Science, 370-375.

Kim, H., 2003, A study on the regional vulnerability assessment and its application for the disaster management, Research Report of National Disaster Management Institute, Korea (in Korean).

Kim, Y., Shin, S., 2009, Developing a risk assessment method for the mitigation of urban disasters, Research Report of Seoul Development Institute (2009-PR-33), Korea (in Korean).

NEMA (National Emergency Management Agency, Korea), 2008-2012, Natural hazard statistics (2008-2012), Natural Disaster Annual Report, Korea. Available at http://www.safekorea.go.kr/dmtd/board/BoardList.jsp?q_board_cd =staidata\&q_menuid=M_NST_SVC_02_04_04 (in Korean).

Remondo, J., Bonachea, J., Cendrero, A., 2008, Quantitative landslide risk assessment and mapping on the basis of recent occurrences, Geomorphology 94, 496-507.

RIMGIS (RIver Management GIS), Han River Flood Control Office, the 
Korean Ministry of Land Infrastructure and Transport (MLIT):

http://www.river.go.kr/index.aspx (in Korean).

Sassa, K., Wang, G., Fukuoka, H., Wang, F., Ochiai, T., Sugiyama, M.,

Sekiguchi, T., 2004, Landslide risk evaluation and hazard zoning for rapid and long-travel landslides in urban development areas, Landslides 3, 221-235.

Scenna, M. J., Cruz, S., 2005, Road risk analysis due to the transportation of chlorine in Rosario city, Reliability Engineering and System Safety 90, 8390.

Statistics Korea, 2010, Population and housing census of 2010, Korea.

Available at http:/ / census.go.kr/hcensus/index.jsp (in Korean).

Thomalla, F., Downing, T., Spanger-siegfried, E., Han, G., Rockstrom, J., 2006, Reducing hazard vulnerability: towards a common approach between disaster risk reduction and climate adaptation, Disasters 30(1), 39-48.

U.S. Department of Energy, 2012, Climate change and infrastructure, urban systems, and vulnerabilities, Technical Report to the U.S. Department of Energy in Support of the National Climate Assessment, USA. Available at http:/ / www.esd.ornl.gov/eess/Infrastructure.pdf

WAMIS (WAter Management Information System), the Korean Ministry of 
Land, Infrastructure and Transport (MLIT):

http://www.wamis.go.kr/ 


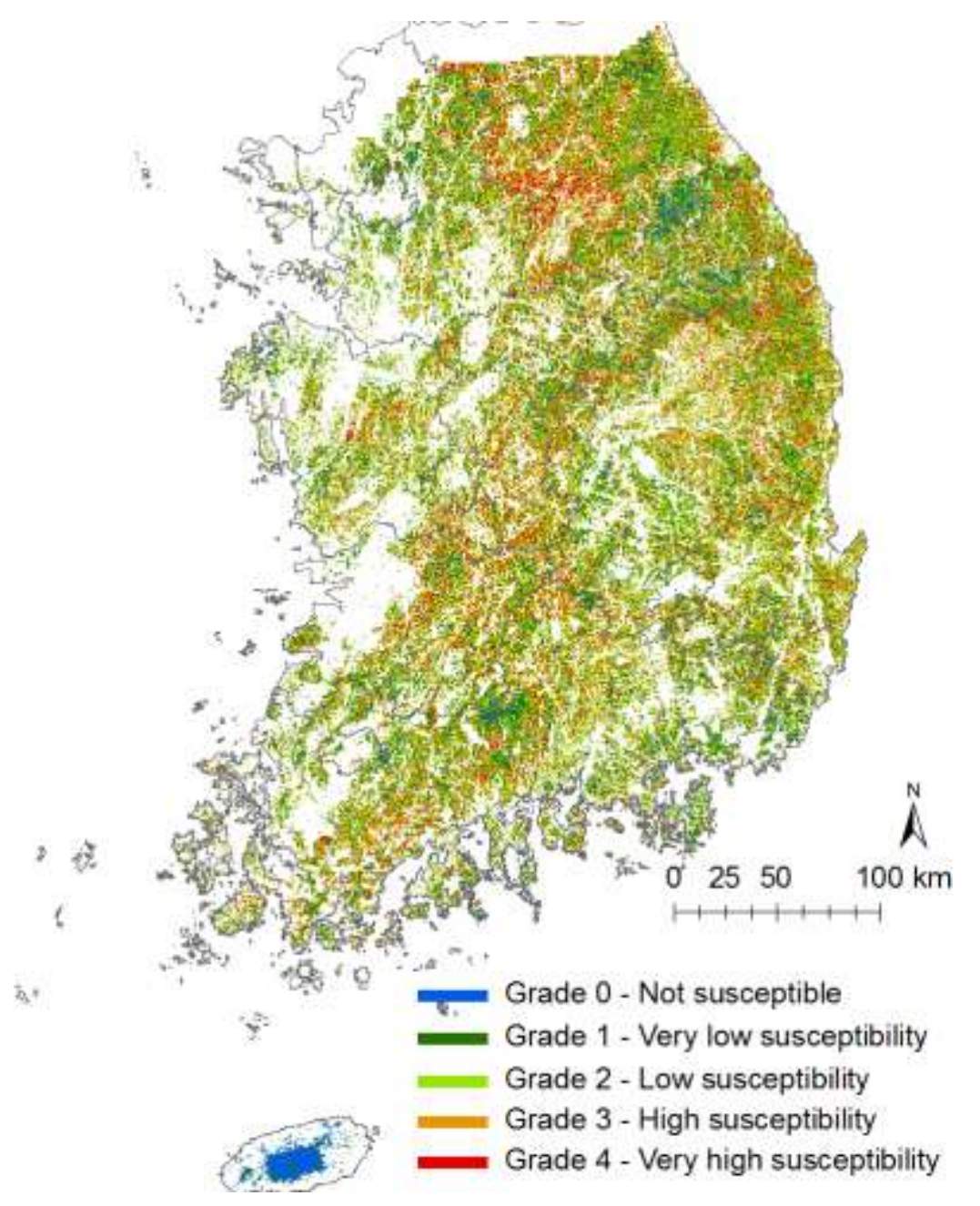

Figure 1. Landslide hazard map of Korea 


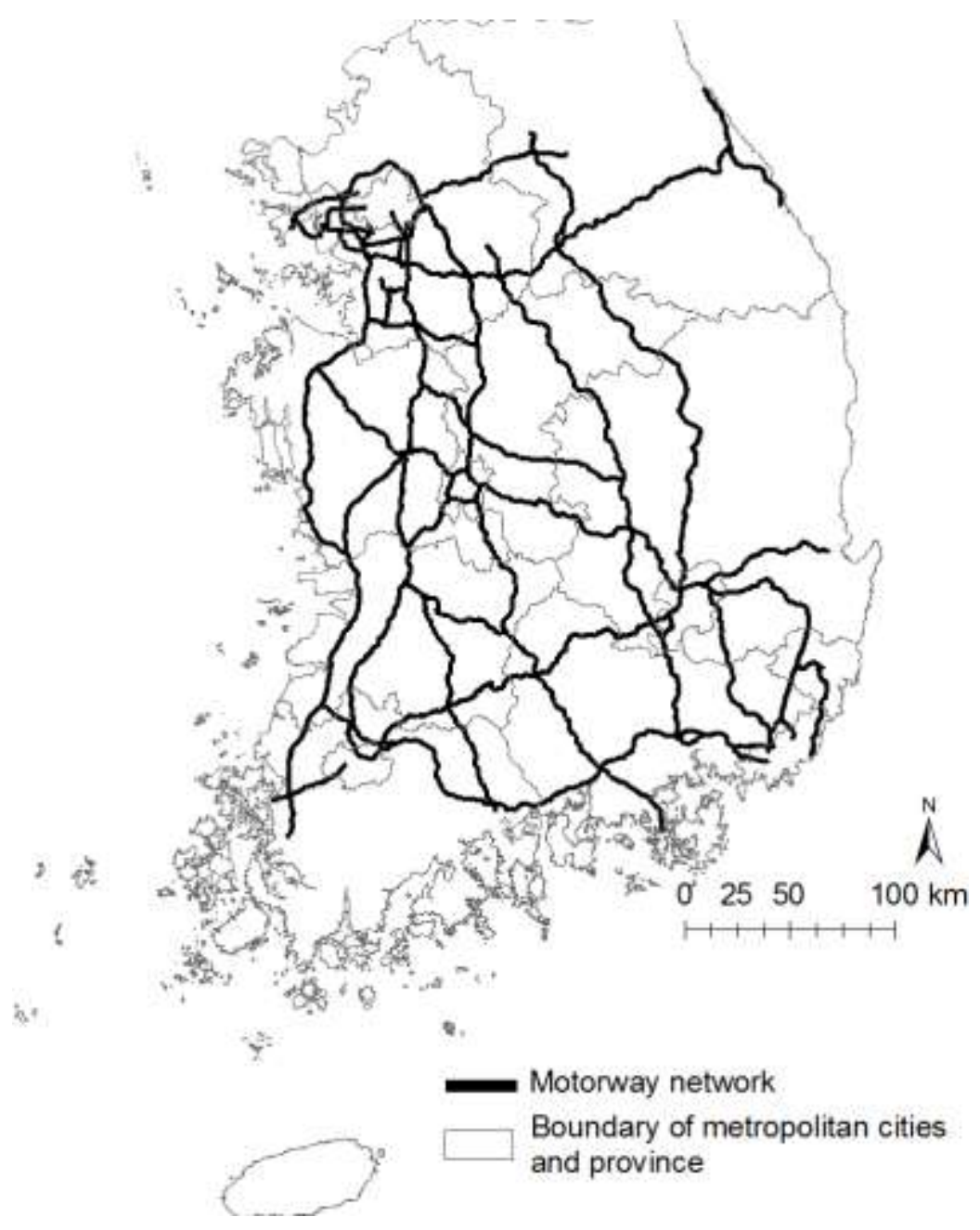

Figure 2. Motorway network of Korea 


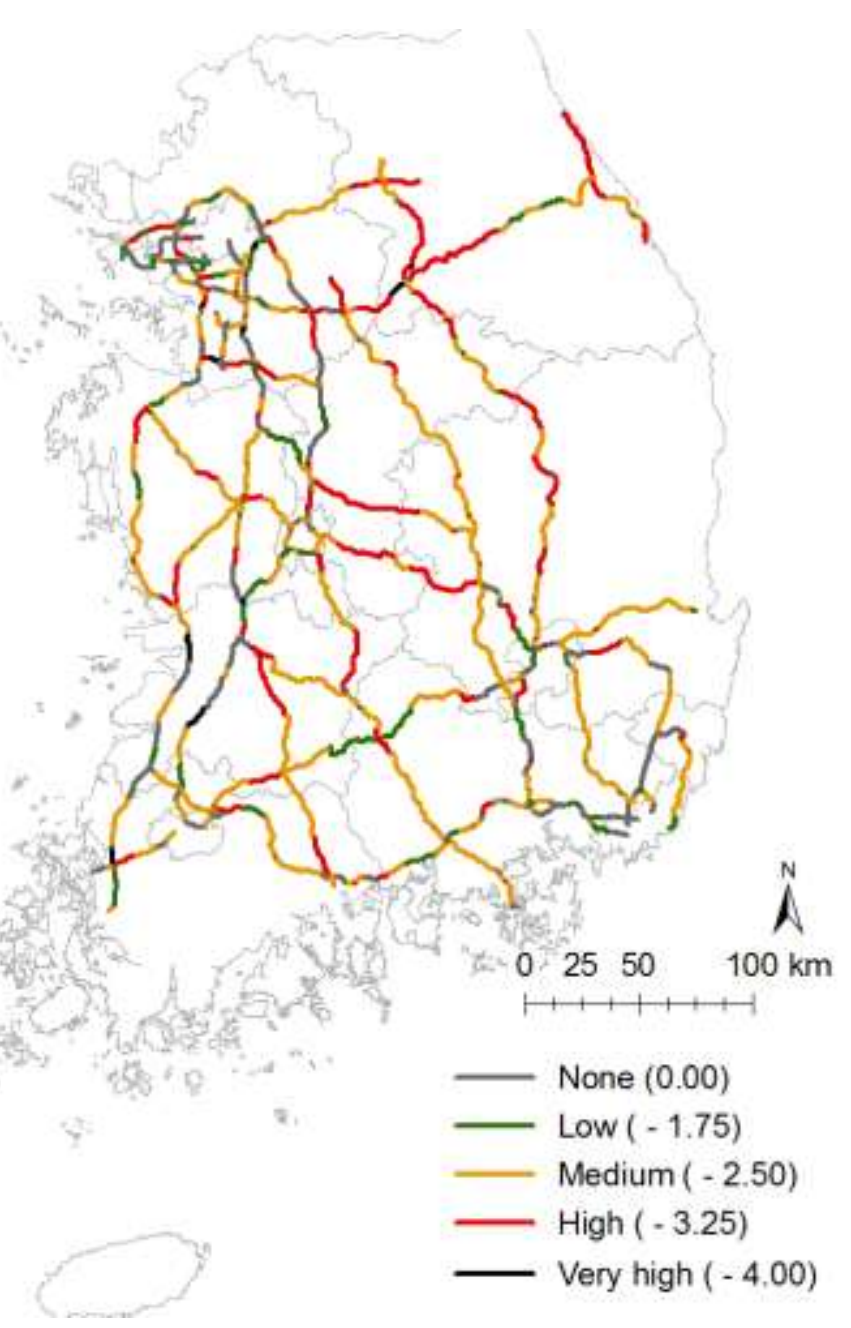

Figure 3. Road risk map of landslide hazard generated by assessing the link length weighted average according to the landslide hazard grades (the value of the WALH index) 


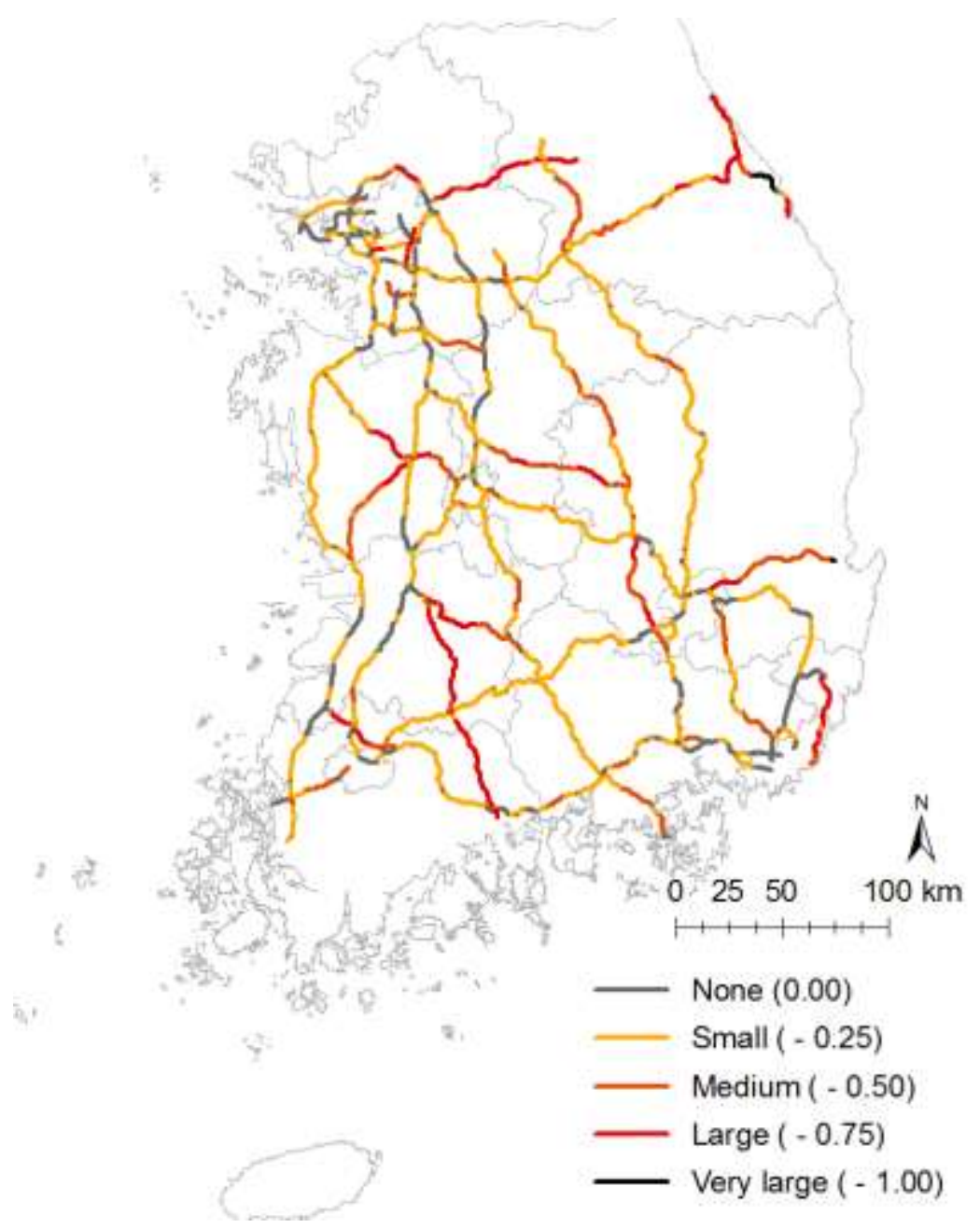

Figure 4. Road risk map for landslide hazard generated by assessing the proportion of link length that is exposed to landslide hazard within a unit link (the value of the PLLH index) 


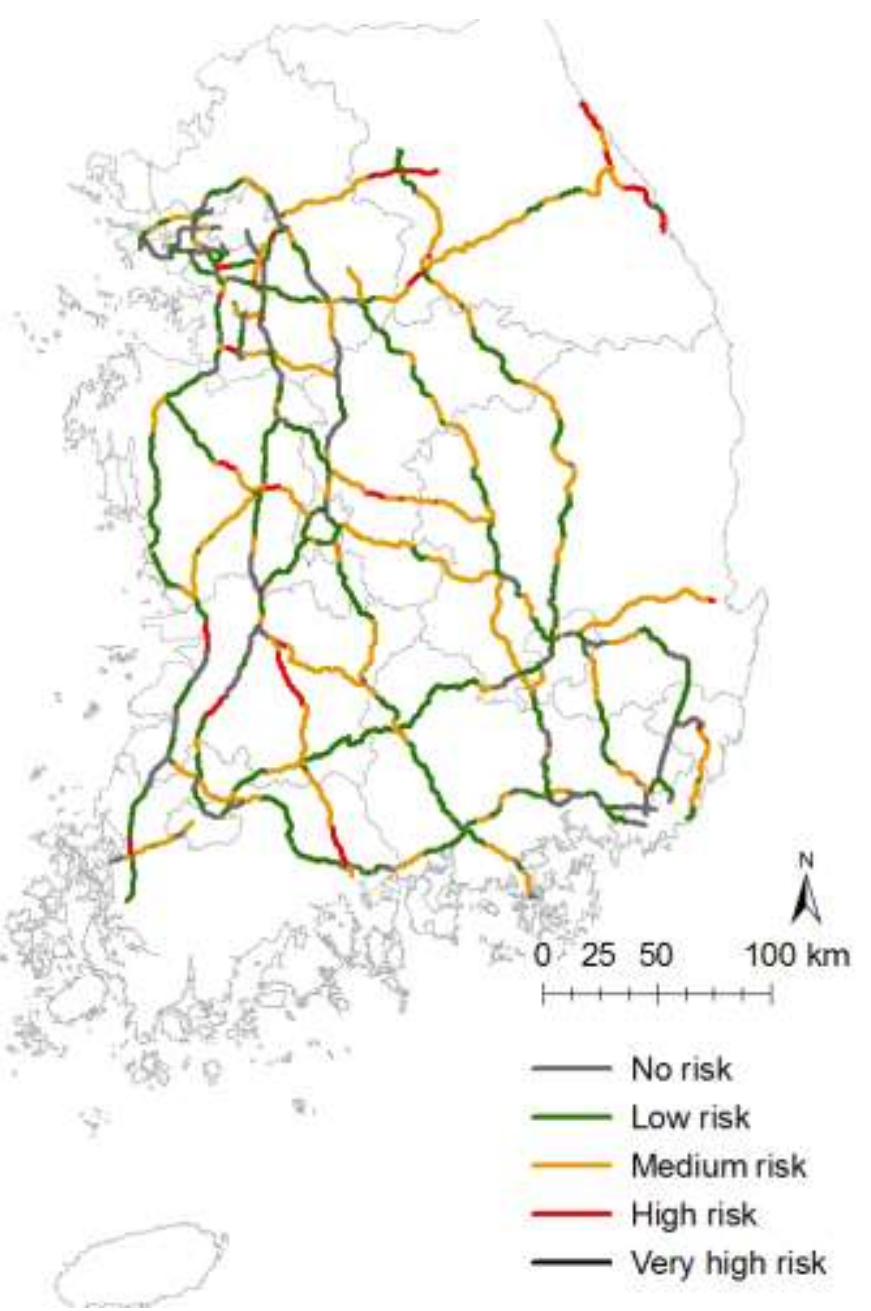

Figure 5. Road risk map for landslide hazard determined using matrix analysis that considered two indices 


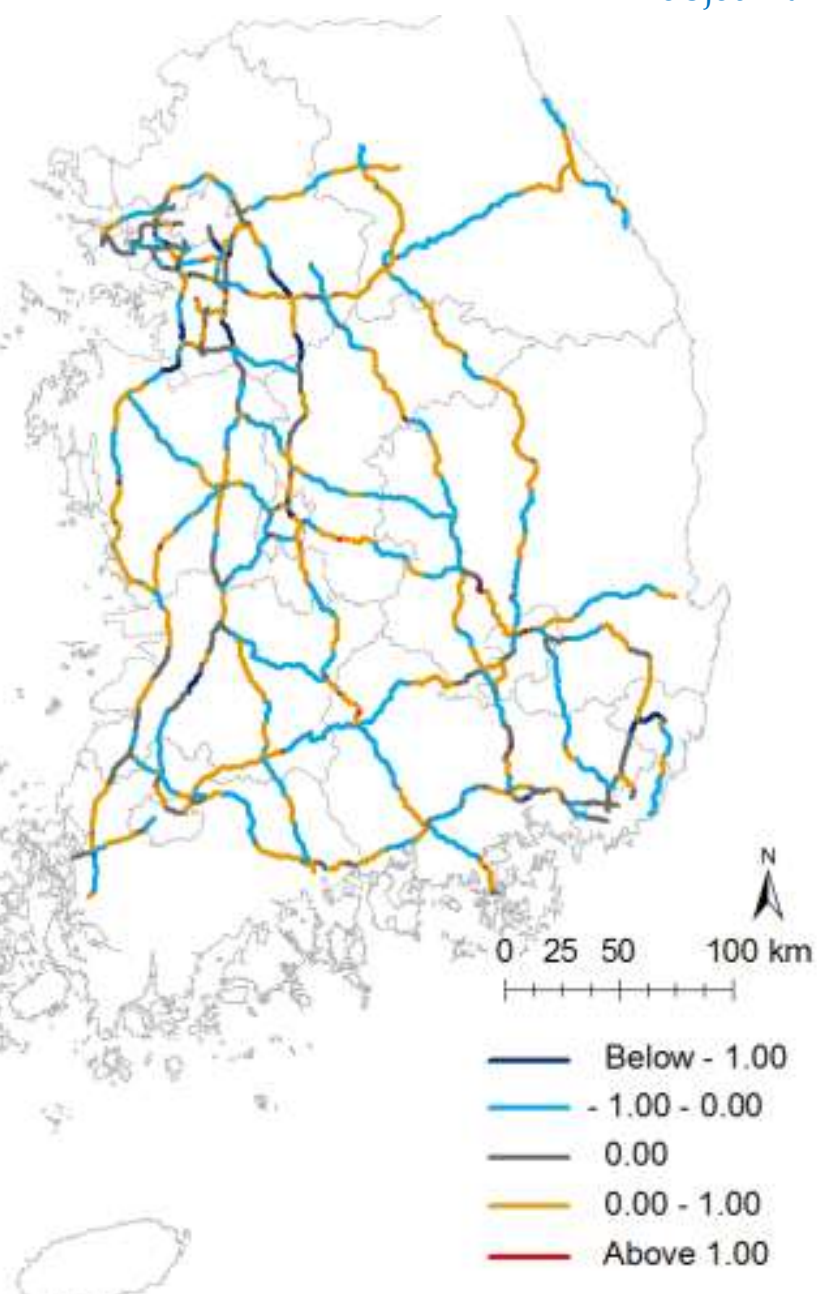

Figure 6. Difference of weighted average of landslide hazard grade between line and polygon data sets for the road network 
Table 1. Amount of damage caused by natural disasters in Korea (NEMA, 2008-2012)

(Units: one hundred million KRW (approximately one hundred thousand USD), \%)

\begin{tabular}{c|r|r|r|r|r}
\hline Year & Typhoon & Heavy rain & Heavy snow & Others & \multirow{2}{*}{ Total } \\
\hline \multirow{2}{*}{2008} & 0.9 & 6.7 & 1.6 & 1.1 & \multirow{2}{*}{10.2} \\
\hline \multirow{2}{*}{2009} & $8 \%$ & $65 \%$ & $15 \%$ & $11 \%$ & \\
\hline \multirow{2}{*}{2010} & - & 254.9 & 12.8 & 31.1 & \multirow{2}{*}{298.8} \\
& 172.5 & 180.8 & 66.3 & 7.2 & \multirow{2}{*}{426.8} \\
\hline \multirow{2}{*}{2011} & 218.3 & 527.6 & 48.0 & 0.3 & \multirow{2}{*}{794.2} \\
\hline \multirow{2}{*}{2012} & $27 \%$ & $66 \%$ & $6 \%$ & $0 \%$ & \\
& $1,003.5$ & 38.4 & 19.6 & 26.7 & \multirow{2}{*}{$1,088.1$} \\
\hline
\end{tabular}

Table 2. Number of grid cells by landslide hazard grade and their proportion 


\begin{tabular}{|c|c|c|c|}
\hline Grades & Landslide hazard & \# of grid cells & Ratio \\
\hline 0 & Not susceptible & $40,192,402$ & $7.3 \%$ \\
\hline 1 & Very low susceptibility & $117,956,374$ & $21.4 \%$ \\
\hline 2 & Low susceptibility & $199,336,367$ & $36.1 \%$ \\
\hline 3 & High susceptibility & $132,801,820$ & $24.0 \%$ \\
\hline 4 & Very high susceptibility & $62,032,941$ & $11.2 \%$ \\
\hline \multicolumn{2}{|r|}{ Total } & $552,319,904$ & $100.0 \%$ \\
\hline
\end{tabular}


Table 3. Matrix assessment using two indices: values of WALH and PLLH

\begin{tabular}{|c|c|c|c|c|c|c|c|}
\hline & & & \multicolumn{5}{|c|}{ Value of WALH Index } \\
\hline & & & None & Low & Medium & High & Very high \\
\hline & & & 0.00 & $1.00-1.75$ & $1.75-2.50$ & $2.50-3.25$ & $3.25-4.00$ \\
\hline \multirow{5}{*}{$\begin{array}{l}\text { Value } \\
\text { of } \\
\text { PLLH } \\
\text { Index }\end{array}$} & None & 0.00 & No risk & & & & \\
\hline & Small & $0.00-0.25$ & & Low & Low & Medium & High \\
\hline & Medium & $0.25-0.50$ & & Low & Medium & Medium & High \\
\hline & Large & $0.50-0.75$ & & Medium & Medium & High & Very high \\
\hline & Very large & $0.75-1.00$ & & High & High & Very high & Very high \\
\hline
\end{tabular}

Table 4. Number of links and ratio determined by classifying value of WALH index

\begin{tabular}{|c|c|c|c|}
\hline \multicolumn{2}{|c|}{ Classification } & \# of links & Ratio \\
\hline None & 0.00 & 375 & $41 \%$ \\
\hline Low & $1.00-1.75$ & 82 & $9 \%$ \\
\hline Medium & $1.75-2.50$ & 282 & $31 \%$ \\
\hline High & $2.50-3.25$ & 155 & $17 \%$ \\
\hline Very high & $3.25-4.00$ & 17 & $2 \%$ \\
\hline \multicolumn{2}{|c|}{ Total } & 911 & $100 \%$ \\
\hline
\end{tabular}


Table 5. Number of links and ratio determined by classifying the value of the PLLH index

\begin{tabular}{|c|c|c|c|}
\hline \multicolumn{2}{|c|}{ Classification } & \# of links & Ratio \\
\hline None & 0.00 & 375 & $41.2 \%$ \\
\hline Small & $0.00-0.25$ & 364 & $40.0 \%$ \\
\hline Medium & $0.25-0.50$ & 104 & $11.4 \%$ \\
\hline Large & $0.50-0.75$ & 64 & $7.0 \%$ \\
\hline Very large & $0.75-1.00$ & 4 & $0.4 \%$ \\
\hline \multicolumn{2}{|c|}{ Total } & 911 & $100.0 \%$ \\
\hline
\end{tabular}

Table 6. Number of links and ratio determined by classifying road risk matrix using two indices

\begin{tabular}{c|r|r}
\hline Classification & \# of links & \multicolumn{1}{c}{ Ratio } \\
\hline No risk & 375 & $41.2 \%$ \\
\hline Low risk & 257 & $28.2 \%$ \\
\hline Medium risk & 232 & $25.5 \%$ \\
\hline High risk & 46 & $5.0 \%$ \\
\hline Very high risk & 1 & $0.1 \%$ \\
\hline Total & 911 & $100.0 \%$ \\
\hline
\end{tabular}


Table 7. State transition matrix for adjusted road links

\begin{tabular}{|c|c|c|c|c|c|c|c|}
\hline & \multicolumn{6}{|c|}{ Polygon data } \\
\hline & & None & Low & Medium & High & Very high & Total \\
\hline \multirow{6}{*}{$\begin{array}{c}\text { Polyline } \\
\text { data }\end{array}$} & None & $\begin{array}{r}351 \\
(38.5 \%) \\
\end{array}$ & $\begin{array}{r}18 \\
(2.0 \%) \\
\end{array}$ & $\begin{array}{r}3 \\
(0.3 \%) \\
\end{array}$ & $\begin{array}{r}3 \\
(0.3 \%) \\
\end{array}$ & $\begin{array}{r}0 \\
(0.0 \%) \\
\end{array}$ & $\begin{array}{r}375 \\
(41.2 \%) \\
\end{array}$ \\
\hline & Low & $\begin{array}{r}30 \\
(3.3 \%) \\
\end{array}$ & $\begin{array}{r}219 \\
(24.0 \%) \\
\end{array}$ & $\begin{array}{r}7 \\
(0.8 \%) \\
\end{array}$ & $\begin{array}{r}1 \\
(0.1 \%) \\
\end{array}$ & $\begin{array}{r}0 \\
(0.0 \%) \\
\end{array}$ & $\begin{array}{r}257 \\
(28.2 \%) \\
\end{array}$ \\
\hline & Medium & $\begin{array}{r}13 \\
(1.4 \%) \\
\end{array}$ & $\begin{array}{r}11 \\
(1.2 \%) \\
\end{array}$ & $\begin{array}{r}194 \\
(21.3 \%) \\
\end{array}$ & $\begin{array}{r}13 \\
(1.4 \%) \\
\end{array}$ & $\begin{array}{r}1 \\
(0.1 \%)\end{array}$ & $\begin{array}{r}232 \\
(25.5 \%)\end{array}$ \\
\hline & High & $\begin{array}{r}2 \\
(0.2 \%) \\
\end{array}$ & $\begin{array}{r}0 \\
(0.0 \%)\end{array}$ & $\begin{array}{r}5 \\
(0.5 \%) \\
\end{array}$ & $\begin{array}{r}36 \\
(4.0 \%) \\
\end{array}$ & $\begin{array}{r}3 \\
(0.3 \%) \\
\end{array}$ & $\begin{array}{r}46 \\
(5.0 \%) \\
\end{array}$ \\
\hline & Very high & $\begin{array}{r}0 \\
(0.0 \%)\end{array}$ & $\begin{array}{r}0 \\
(0.0 \%)\end{array}$ & $\begin{array}{r}0 \\
(0.0 \%)\end{array}$ & $\begin{array}{r}0 \\
(0.0 \%)\end{array}$ & $\begin{array}{r}1 \\
(0.1 \%)\end{array}$ & $\begin{array}{r}1 \\
(0.1 \%)\end{array}$ \\
\hline & Total & $\begin{array}{r}396 \\
(43.5)\end{array}$ & $\begin{array}{r}248 \\
(27.2 \%)\end{array}$ & $\begin{array}{r}209 \\
(22.9 \%)\end{array}$ & $\begin{array}{r}53 \\
(5.8 \%)\end{array}$ & $\begin{array}{r}5 \\
(0.5 \%)\end{array}$ & $\begin{array}{r}911 \\
(100 \%)\end{array}$ \\
\hline
\end{tabular}

